\title{
Primer registro del dinoflagelado invasor Ceratium furcoides (Levander) Langhans 1925 en la Argentina y su distribución en el área de influencia del Embalse Yacyretá (río Paraná, Argentina-Paraguay)
}

\author{
Norma Meichtry de Zaburlin ${ }^{1, *}$, Andrés Boltovskoy ${ }^{2}$, Cecilia Costigliolo Rojas ${ }^{1}$ \\ y Romina M. Rodriguez ${ }^{1}$ \\ ${ }^{1}$ Facultad de Ciencias Exactas, Químicas y Naturales, Universidad Nacional de Misiones. Rivadavia 2370, \\ N3300LDX, Posadas, Misiones, Argentina. \\ 2 División Ficología “Dr. Sebastián A. Guarrera”, Museo de la Plata, La Plata, Buenos Aires, Argentina. \\ * Autor responsable de la correspondencia: meichtry4@ hotmail.com
}

Recibido: 03/10/2013

Aceptado: 20/03/2014

\begin{abstract}
First record of the invasive dinoflagellate Ceratium furcoides (Levander) Langhans 1925 in Argentina and its distribution in the influence area of the Yacyretá Reservoir (Paraná River, Argentina-Paraguay)

In this study, we report for the first time the presence of Ceratium furcoides in Argentina, in the area of influence of the Yacyretá Reservoir, Paraná River. Though with low population densities (up to 15 cells/ ml), the species rapidly spread and developed in diverse trophic conditions, so it can be considered as established in the area. Being an invasive species in the region, it probably will spread to the lower sections of the Paraná River in the near future, as a result of passive dispersal through the main channel.
\end{abstract}

Key words: Ceratium furcoides, first record, invader species, subtropical reservoir.

\section{RESUMEN}

Primer registro del dinoflagelado invasor Ceratium furcoides (Levander) Langhans 1925 en la Argentina y su distribución en el área de influencia del Embalse Yacyretá (río Paraná, Argentina-Paraguay)

En el presente trabajo se reporta por primera vez la presencia de Ceratium furcoides en Argentina, en el área de influencia del embalse Yacyretá, río Paraná. Si bien con baja densidad poblacional (máximo de $15 \mathrm{ind} / \mathrm{ml}$ ), la especie se dispersó rápidamente y se desarrolló en diferentes condiciones tróficas, por lo que puede considerarse como establecida en el área. Siendo una especie invasora en la región, es probable que en un futuro cercano se extienda a los tramos inferiores del río Paraná como consecuencia de una dispersión pasiva a través del cauce principal.

Palabras clave: Ceratium furcoides, primer registro, especie invasora, embalse subtropical.

\section{INTRODUCCIÓN}

Entre los dinoflagelados de agua dulce Ceratium Schrank, 1793 es uno dos los géneros más importantes en cuanto a su incidencia en la ecología de los cuerpos de agua. Se trata de un género subcosmopolita pantropical que no posee representantes que produzcan toxicidad, aunque es capaz de ocasionar intensas floraciones. Si bien su presencia se registra en cuerpos de agua de todos los niveles tróficos (Popovský \& Pfiester 1990; Pollingher, 1987) se ve favorecido por las aguas 
ricas en nutrientes, (Heaney et al., 1988; Ginkel et al., 2007; Santos-Wisniewski et al., 2007; Matsumura-Tundisi et al., 2010; Bustamante Gil et al., 2012).

Para ambientes de agua dulce Popovský \& Pfiester (1990) reportan 6 especies de Ceratium. Entre ellas, solamente Ceratium hirundinella y C. furcoides son de hallazgo habitual. La amplia distribución del género era rica en menciones para el hemisferio Norte y muy pobre para el hemisferio Sur (Margalef, 1983). Esta situación se mantuvo hasta la década de 1990, en que el género comenzó a expandirse en Sudamérica y África (Guerrero \& Echenique, 1997; Ginkel et al., 1999; Boltovskoy et al., 2003, 2013; Silva et al., 2012). Entre estas especies, $C$. hirundinella (O. F. Müller) Schrank ha sido registrada en altas densidades en cuerpos de agua eutróficos en los distintos continentes, en países tales como Argentina (Claps \& Ardohain, 2007; Bazán et al., 2007), Australia (Whittington et al., 2000), España (Pérez-Martínez \& Sánchez-Castillo, 2001) y Sudáfrica (Hart, 2007). Las especies del género Ceratium han sido reconocidas como invasoras en ambientes dulceacuícolas en Sudamérica (Boltovskoy, 2005; Silva et al., 2012), sus florecimientos están asociados con cambios ambientales y como consecuencia directa o indirecta de su proliferación se han registrado casos de mortandad de peces en diversos países (Nicholls et al., 1980; Bazán et al., 2007; Canadá y Argentina respectivamente). Particularmente, en Argentina $C$. hirundinella fue hallado por primera vez en 1963, en lagos Andino Patagónicos con muy escasos ejemplares (Thomasson, 1963). Vuelve a registrarse en Argentina en la década de 1990, en Neuquén, Río Negro y Buenos Aires (Guerrero \& Echenique, 1997), y ya en forma invasiva realiza una rápida expansión ampliando su área de distribución hacia embalses y ríos del centro y norte del país (Boltovskoy et al., 2003; Boltovskoy, 2005). Su presencia fue reportada para las provincias de Córdoba, San Luis, Catamarca, Salta, ya sea con escasos individuos y/o constituyendo floraciones (Casco et al., 2002; Fernández-Belmonte et al., 2003; Mac Donagh et al., 2005; Silverio et al., 2009). Hasta el presente no existían datos sobre la ocurrencia de Ceratium furcoides (Levander) Langhans 1925 en el país. No obstante, en Brasil la especie ha sido registrada recientemente en varias publicaciones. Por primera vez en el embalse de la represa hidroeléctrica de Furnas, en el estado de Minas Gerais (Santos-Wisniewski et al., 2007; Silva et al., 2012) y posteriormente en el embalse urbano de Billings, el cual recibe un importante aporte de efluentes domésticos e industriales de la región metropolitana de San Pablo, causando un deterioro de la calidad del agua (MatsumuraTundisi et al., 2010). Asimismo, esta especie fue hallada en una serie de embalses y ríos de la zona semiárida del NE Brasil (Oliveira et al., 2011) y en embalses del estado de San Pablo, i.e. Guarapiranga, Jaguari y Barra Bonita (Amazonas et al., 2012) y en la región sur del país, en los estados de Paraná (río Cachoeira) y Rio Grande do Sul (río Uruguay y Jacuí) (Cavalcante et al., 2013).

En el presente trabajo se reporta por primera vez la presencia de Ceratium furcoides en la Argentina, en el área de influencia del embalse Yacyretá (río Paraná, Argentina-Paraguay), la cual es considerada una especie invasora para la región (Boltovskoy et al., 2013).

\section{MATERIALES Y MÉTODOS}

Ceratium furcoides se identificó a partir de los muestreos de fitoplancton realizados en el marco del monitoreo del Programa de Calidad del Agua del Embalse Yacyretá. La Represa Yacyretá está localizada al norte de la provincia de Corrientes $\left(27^{\circ} 28^{\prime} \mathrm{S}\right.$ y $\left.56^{\circ} 44^{\prime} \mathrm{O}\right)$ sobre el río Alto Paraná, unos $80 \mathrm{~km}$ aguas abajo de la ciudad de Posadas, Misiones, Argentina. Es un embalse de gran tamaño, tiene una superficie de $1600 \mathrm{~km}^{2}$, una longitud de cauce de $342 \mathrm{~km}$, un ancho máximo de $30 \mathrm{~km}$, un volumen de $21000 \mathrm{hm}^{3}$, profundidades medias de 7-8 m y máximas de $34 \mathrm{~m}$. Este embalse se caracteriza por su corto tiempo de residencia del agua, entre 17 y 23 días dependiendo del caudal ingresante (Meichtry de Zaburlin et al., 2013a). Para el presente estudio, las muestras cualitativas se recolectaron con red de plancton de 25 micrones de abertura de malla y las cuantitativas con una botella tipo Van Dorn. Los 
muestreos se realizaron con frecuencia mensual en nueve estaciones (Fig. 1) distribuidas en las distintas secciones del embalse: estación de entrada o fluvial (E1), estación de transición (E2), estaciones lacustres o del embalse (E3MD, E3C, E3MI, E4MD, E4MI, E-Bahía Santa María), estación de salida, aguas abajo de la presa (E5) y con una frecuencia trimestral en cinco estaciones ubicadas en los brazos laterales (subembalses de los arroyos Pindapoy, Garupá, Mártires, Itaembé y Yacarey), desde febrero de 2011, cuando se alcanzó la cota máxima definitiva de $83 \mathrm{msnm}$, hasta julio de 2013. Los recuentos se realizaron según la técnica de Utermöhl (1958).

\section{RESULTADOS Y DISCUSIÓN}

Ceratium furcoides se detectó por primera vez en el río Alto Paraná en octubre de 2012 en dos tributarios del embalse Yacyretá (arroyos Pindapoy y Garupá), los cuales se vieron fuertemente modificados como consecuencia del llenado del embalse a su nivel máximo de operación a $83 \mathrm{msnm}$ en febrero de 2011. Estos arroyos ampliaron su área de inundación ocupando su valle, transformándose así en subembalses laterales, con mayor tiempo de residencia del agua, disminución en su velocidad de escurrimiento y cambios en la calidad del agua (Meichtry de Zaburlin et al., 2013a).

En los meses sucesivos se observó una rápida colonización y el establecimiento progresivo de la especie en las distintas secciones del em- balse, siendo el registro más austral la transecta ubicada en el río Paraná a la altura de la localidad de Itá Ibaté en la provincia de Corrientes. En el mes de julio de 2013 C. furcoides se registró en todas las estaciones del cuerpo principal del embalse y subembalses estudiados (Fig. 1). La mayor abundancia se registró en el arroyo Pindapoy en abril de 2013, alcanzando una concentración de $15 \mathrm{ind} / \mathrm{ml}$ y en otros tributarios afectados por el vertido de efluentes cloacales y domiciliarios como el arroyo Mártires con 7 ind/ml.

Las aguas del embalse Yacyretá se caracterizan por su baja conductividad (mín: 42, máx: $61 \mu \mathrm{S} / \mathrm{cm}$ ), pH neutro a levemente alcalino (mín: 7.02, máx: 7.9), temperaturas medias entre 22 y $24^{\circ} \mathrm{C}$, moderada transparencia del agua (mín: 40 , máx: $380 \mathrm{~cm})$, alta concentración de oxígeno disuelto (valores medios mayores a $8 \mathrm{mg} / \mathrm{l}$ ), baja concentración de nutrientes (fósforo total: valores medios entre 16 y $19 \mu \mathrm{g} / \mathrm{l})$ y condiciones oligotróficas en el cuerpo principal (Meichtry de Zaburlin et al., 2013a) y mesotróficas a eutróficas en algunos subembalses laterales como el arroyo Mártires, que recibe una importante descarga de efluentes cloacales y domiciliarios, con valores elevados de nutrientes (fósforo total: mín: 44, máx: $380 \mu \mathrm{g} / \mathrm{l})$, menor concentración de oxígeno disuelto (mín: 5.1, máx: $5.6 \mathrm{mg} / \mathrm{l}$ ) y transparencia del agua (mín: 50, máx: $150 \mathrm{~cm}$ ).

Ceratium furcoides es un dinoflagelado relativamente grande, de entre 123 y $322 \mu \mathrm{m}$ de longitud y un transdiámetro de 28-42 (56) $\mu \mathrm{m}$ (Popovský \& Pfiester, 1990). Tiene un cuerno epitecal

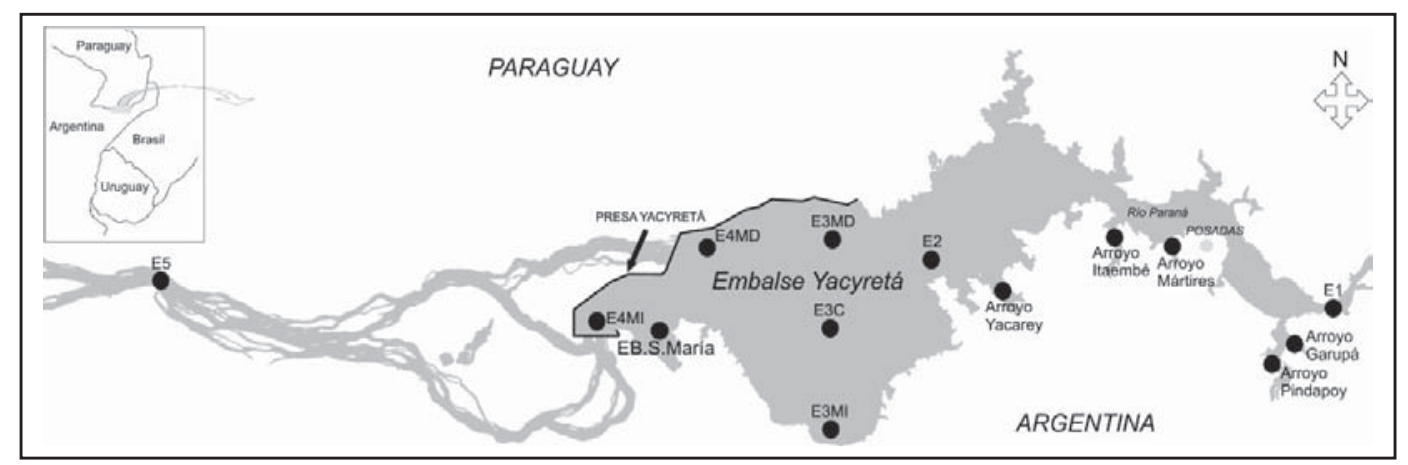

Figura 1. Mapa de distribución de Ceratium furcoides y estaciones de muestreo. E: estación, MD: margen derecha, MI: margen izquierda, C: centro. Distribution of Ceratium furcoides and sampling sites. E: station, MD: right margin, MI: left margin, C: center. 
grande y dos, raramente tres, en la hipoteca (Bustamante Gil et al., 2012). Esta especie se diferencia de $C$. hirundinella por la configuración de las placas apicales. Mientras que esta última presenta sus cuatro placas apicales alcanzando el ápice de la célula, en $C$. furcoides solo las tres primeras placas apicales $\left(1^{\prime}, 2^{\prime}\right.$ y $\left.3^{\prime}\right)$ llegan hasta el ápice, siendo la cuarta apical (4') mucho más corta; al mismo tiempo existen diferencias, no siempre evidentes, en el aspecto de la base de sus epitecas, siendo acampanada en $C$. hirundinella y casi cónica en $C$. furcoides (Calado \& Larsen, 1997). Nuestro material de C. furcoides (Fig. 2) muestra ambas características distintivas de la especie y sus medidas fluc- túan entre 146-212 $\mu \mathrm{m}$ de longitud y 37-45 $\mu \mathrm{m}$ de transdiámetro, entrando dentro del rango de medidas establecidas para la especie.

Si bien por el momento no es posible precisar el origen de la introducción de la especie en la Argentina, es de suponer que estaría asociado a una dispersión pasiva a través del flujo de agua a partir de tributarios del río Paraná Superior (Brasil) donde se ha registrado la presencia de $C$. furcoides (Santos-Wisniewski et al., 2007; MatsumuraTundisi et al., 2010; Cavalcante et al., 2013). Sin embargo no se pueden descartar como factores de su dispersión el transporte de sus estados de resistencia (quistes) por las aves, insectos acuáticos o la intervención antrópica (Kristiansen, 1996).
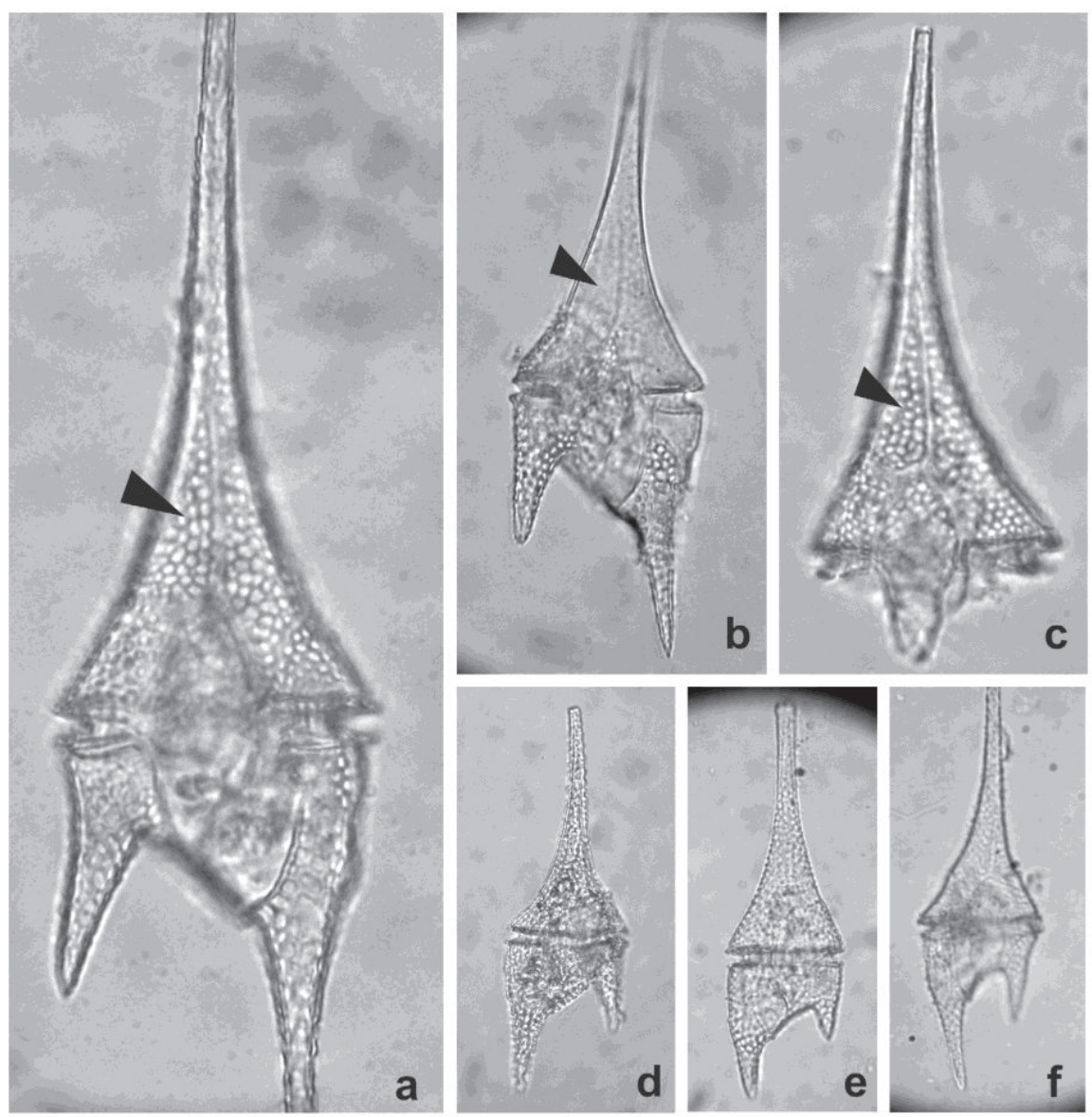

Figura 2. Ceratium furcoides del embalse Yacyretá: a, b. Vista ventral; c. Vista ventral de la epiteca. (Las flechas señalan la corta cuarta placa apical $\left(4^{\prime}\right)$ característica de la especie). d, e, f. Vista dorsal. Ceratium furcoides from Yacyretá reservoir: a, b. Ventral view. c. Ventral view of the epitheca. (The arrowheads indicate the short fourth apical plate (4') distinctive of the species). $d$, e, $f$. Dorsal views. 
En América del Sur, el emplazamiento de embalses para generación de energía eléctrica, abastecimiento de agua e irrigación han aumentado notablemente en las últimas décadas (Nogueira et al., 2005; Meichtry de Zaburlin et al., 2010; Meichtry de Zaburlin et al., 2013b) favoreciendo el desarrollo de especies invasoras. La invasión de especies exóticas puede producir cambios irreversibles en la composición y estructura de los ecosistemas (Silva et al., 2012). Aunque hasta el presente no existe una explicación coherente, la instalación del género Ceratium en Sudamérica podría asociarse a cambios climáticos, a modificaciones en el régimen hidrológico y la calidad de las aguas.

Los florecimientos de $C$. furcoides pueden ser perjudiciales para la fauna íctica y la comunidad de invertebrados, debido al colapso de las floraciones, cuya posterior descomposición puede provocar el agotamiento del oxígeno disuelto (Matsumura-Tundisi et al., 2010). Por otro lado, la alta densidad poblacional que puede alcanzar la especie suele representar un serio problema para el tratamiento del agua destinada al consumo humano.

De los datos analizados en este trabajo podemos concluir que la aparición de la $C$. furcoides en Yacyretá podría relacionarse con un aumento del tiempo de residencia y estabilidad de la columna de agua, y particularmente en los subembalses laterales, con la disponibilidad de nutrientes mayor que la registrada en el cauce principal del río Paraná (Meichtry de Zaburlin et al., 2013a). Una creciente ocurrida en el embalse en el mes de julio de 2013 podría explicar la dispersión de la especie en los diferentes sitios ubicados aguas abajo. Sin embargo, en nuestro caso, las densidades registradas hasta el presente fueron bajas y al parecer aún no han afectado las condiciones ambientales y de otras comunidades.

Cabe resaltar que la mencionada especie se dispersó rápidamente y se desarrolló en distintos ambientes y secciones del embalse con diferentes niveles tróficos, lo que la caracteriza como una especie exótica invasora de importancia en la región. En función de los antecedentes mencionados, la especie puede considerarse como establecida en el área, y es probable que en un futuro cercano se registre su presencia en tramos infe- riores del río Paraná. Dado que las floraciones de la especie pueden tener un incidencia directa sobre la calidad del agua para consumo humano y sobre la estructura de las comunidades acuáticas, se sugiere a los organismos de aplicación el monitoreo de la presencia de la especie para mitigar sus posibles impactos.

\section{REFERENCIAS}

AMAZONAS, D., M. do C. CARVALHO, M. MORANDINI \& M. C. LAMPARELLI. 2012. Ocorrência de Ceratium furcoides (Levander) Langhans 1925 em Reservatórios do Estado de São Paulo, SP-Brasil. In: XIV Congresso Brasileiro de Ficologia. June 17-21, 2012. João Pessoa, Brasil. 29.

BAZÁN, R., N. LARROSA, A. COSSAVELLA, F. MONARDE, S. OVIEDO ZABALA, A. RODRÍGUEZ \& F. BUSSO. 2007. Mortandad de peces en el embalse Los Molinos asociados a florecimientos de Ceratium hirundinella. Instituto Superior de Investigación y Servicios de Recursos HídricosUNC. Dirección Provincial de Agua y Saneamiento. Aguas Cordobesas S.A.

BOLTOVSKOY, A. 2005. Ceratium hirundinella Schrank: Un dinoflagelado invasor en lagos y embalses. Simposio en XXX Jornadas Argentinas de Botánica. Noviembre 6 al 10, 2005. Santa Fe, Argentina. Boletín de la Sociedad Argentina de Botánica, 40: 9.

BOLTOVSKOY, A., R. ECHENIQUE \& J. M. GUERRERO. 2003. Colonización de limnotopos de Argentina por Ceratium hirundinella (Dinophyceae). Boletín de la Sociedad Argentina de Botánica, 38(Supl): 148.

BOLTOVSKOY, A., R. ECHENIQUE \& J. M. GUERRERO. 2013. Sucesivas invasiones de especies de Ceratium (Dinophyceae) en Sudamérica: Un proceso que lleva dos décadas. Boletín de la Sociedad Argentina de Botánica, 48(Supl): 27.

BUSTAMANTE GIL, C., J. J. RAMIREZ RESTREPO, A. BOLTOVSKOY, \& A. VALLEJO. 2012. Spatial and temporal change characterization of Ceratium furcoides (Dinophyta) in the equatorial reservoir Riogrande II, Colombia. Acta Limnologica Brasiliensia, 24(2): 207-219.

CALADO, A. J. \& J. LARSEN. 1997. On the identity of the type species of the genus Ceratium 
Schrank (Dinophyceae), with notes on $C$. hirundinella. Phycologia, 36: 500-505.

CASCO, M., M. MAC DONAGH \& M. CLAPS. 2002. Long-term study of plankton in Río Tercero Reservoir (Argentine) in relation to a nuclear power plant operation. Verhandlungen des Internationalen Verein Limnologie, 28: 1027-1031.

CAVALCANTE, K. P., J. C. ZANOTELLI, C. C. MÜLLER, K. D. SCHERER, J. K. FRIZZO, T. A. V. LUDWIG \& L. S. CARDOSO. 2013. First record of invasive Ceratium (Schrank, 1973) species (Dinophyceae) in Southern Brazil, with notes on their dispersive patterns in Brazilian environments. Check List, 9(4): 862-866.

CLAPS, M., \& D. ARDOHAIN. 2007. Zooplankton characterization in a subtropical reservoir (Córdoba, Argentina) during the period 1996-2005. Plankton Symposium IV. Journal of Biology, 2: 108.

FERNANDEZ-BELMONTE, M. C., Z. E. FURLAN, M. M. HELLMERS \& E. N. OCAMPO. 2003. Estudio preliminar para el diagnóstico ambiental del dique San Felipe. Boletín de la Sociedad Argentina de Botánica, 38(Supl.): 255.

GINKEL, C. E. van, B. C. HOHLS, \& E. VERMAAK. 1999. A Fish kill in the Hartbeespoort Dam, South Africa, October 1999, Internal IWQS Report No. N/A210/02/DEQ/4299. http://www. dwaf.gov.za/IWQS/reports/carin/fishkill1/Fish \% 20kill1_1999.htm

GINKEL, C. E. van, H. CAO, F. RECKNAGEL, \& S. du PLESSIS, S. 2007. Forecasting of dinoflagellate blooms in warm-monomictic hypertrophic reservoirs in South Africa by means of rule-based agents. Water SA, 33: 532-538. (http://www.wrc. org.za).

GUERRERO, J. \& R. ECHENIQUE. 1997. Ceratium hirundinella blooms in Argentine reservoirs. Harmful Algae News, 16: 3.

HART, R. C. 2007. Temporal Dynamics and spatial perspectives of contemporary blooms of the dinoflagellate Ceratium in a subtropical South African Reservoir. In: Plankton Symposium IV. Journal of Biology, 2: 159.

HEANEY, S. I., J. W. G. LUND, H. M. CANTER \& K. GRAY. 1988. Population dynamics of Ceratium spp. in three English lakes, 1945-1985. Hydrobiologia, 161: 133-148.

KRISTIANSEN, J. 1996. Dispersal of freshwater algae. Hydrobiologia, 336(1-3): 151-157.
MAC DONAGH, M. E., M. A. CASCO, \& M. C. CLAPS. 2005. Colonization of a Neotropical Reservoir (Córdoba, Argentina) by Ceratium hirundinella (O. F. Muller) Bergh. Annales de LimnologieInternational Journal of Limnology, 41(4): 291299.

MARGALEF, R. 1983. Limnología. Omega. Barcelona. España.

MATSUMURA-TUNDISI, T., J. G. TUNDISI, A. P. LUZIA, \& R. M. DEGANI. 2010. Occurrence of Ceratium furcoides (Levander) Langhans 1925 bloom at the Billings Reservoir, São Paulo State, Brazil. Brazilian Journal of Biology, 70(3): 825-829.

MEICHTRY DE ZABURLIN, N., J. G. PESO, G. GARRIDO \& R. E. VOGLER. 2010. Sucesión espacio-temporal del plancton y bentos en periodos posteriores al llenado del Embalse Yacyretá (Río Paraná, Argentina-Paraguay). Interciencia, 35(12): 897-904.

MEICHTRY DE ZABURLIN, N., J. PESO \& P. ARAYA. 2013a. Humedales del Embalse de Yacyretá y ambientes asociados (pág: 113-122) En: Inventario de los humedales de Argentina. Sistemas de paisajes de humedales del Corredor Fluvial Paraná-Paraguay, Proyecto GEF 4206, PNUD/ARG/10/003. ISBN 978-987-29340-0-2. Ed. L. Benzaquen et al. $1^{\text {a }}$ Edición Buenos Aires.

MEICHTRY DE ZABURLIN, N., R. E. VOGLER, V. M. LLANO \& I. S. M. MARTENS. 2013b. Fitoplancton del embalse Yacyretá (ArgentinaParaguay) a una década de su llenado. Revista Mexicana de Biodiversidad, 84(1): 225-239.

NICHOLLS, K. M, W. KENNEDY \& C. HANNET. 1980. Fish kill Heart Lake, Otario, associated with the colapse of a masive population of Ceratium hirundinella (Dinophyceae). Freshwater Biology, 10(6): 553-561.

NOGUEIRA, M. G., A. JORCIN, N. C. VIANNA \& Y. C. T. BRITTO. 2005. Reservatórios em cascata e os efeitos na limnologia e organização das comunidades bióticas (fitoplancton, zooplancton e zoobentos) um estudo de caso no rio Paranapanema. In: Ecologia de reservatórios: impactos potenciais, ações de manejo e sistema em cascata. Org. M. G. Nogueira, R. Henry \& A. Jorcin. São Carlos. Brazil. RiMa: 83-125.

OLIVEIRA, H. S. B., A. N. MOURA, \& M. K. CORDEIRO. 2011. First record of Ceratium Schrank, 1973 (Dinophyceae: Ceratiaceae) in 
freshwater ecosystems in the semiarid region of Brazil. Check List, 7: 626-628.

PÉREZ-MARTÍNEZ, C. \& P. SÁNCHEZ CASTILLO. 2001. Temporal occurrence of Ceratium hirundinella in Spanish reservoirs. Hydrobiologia, 452: 101-107.

POLLINGHER, U. 1987. Freshwater ecosystems. In: The Biology of Dinoflagellates. Taylor, F. J. R. (Ed). Blackwell, Oxford.

POPOVSKÝ, J. \& L. A. PFIESTER. 1990. Süsswasserflora von Mitteleuropa. 6. Dinophyceae (Dinoflagellida). Ettl, H. et al. (Ed) Gustav Fischer, Jena.

SANTOS-WISNIEWSKI, M. J., L. C. SILVA, I. C. LEONE, R. LAUDARES-SILVA \& O. ROCHA. 2007. First record of the occurrence of Ceratium furcoides (Levander) Langhans 1925, an invasive species in the hydroelectricity power plant Furnas Reservoir, MG, Brazil. Brazilian Journal of Biology, 67(4): 791-793.

SILVA, L. C., I. C. LEONE, M. J. SANTOS-WISNIEWSKI, A. C. PERET, \& O. ROCHA. 2012.
Invasion of the dinoflagellate Ceratium furcoides (Levander) Langhans 1925 at tropical reservoir and its relation to environmental variables. Biota Neotropica., 12(2): 93-100.

SILVERIO, M. J., G. MONTAÑEZ, E. FRA, M. SARACHO, M. ARJONA, S. AMAYA \& B. TRACANNA. 2009. Variación poblacional de Ceratium hirundinella(Dinophyceae) en embalses eutróficos de Catamarca (Argentina) y su relación con parámetros ambientales. Huayllu-Bios, 3: 13-31.

THOMASSON, K. 1963. Araucanian Lakes. Acta Phytogeographica Suecica, 47: 1-139.

UTERMOHL, H. 1958. Zur vervolkommung der quantitativen phytoplankton methodik. Mitteilungen Internationale Vereiningung fuer Theoretische und Angewandte Limnologie, 9: 1-38.

WHITTINGTON, J., B. SHERMAN, D GREEN \& R. L OLIVER. 2000. Growth of Ceratium hirundinella in a subtropical Australian reservoir: the role of vertical migration. Journal of Plankton Research., 22(6): 1025-1045. 